\title{
The Astrophysical Implications of Dust Formation During The Eruptions of Hot, Massive Stars
}

\author{
C. S. Kochanek ${ }^{1,2}$
}

\begin{abstract}
Dust formation in the winds of hot stars is inextricably linked to the classic eruptive state of luminous blue variables (LBVs) because it requires very high mass loss rates, $\dot{M} \gtrsim$ $10^{-2.5} M_{\odot} /$ year, for grains to grow and for the non-dust optical depth of the wind to shield the dust formation region from the true stellar photosphere. Thus, dusty shells around hot stars trace the history of "great" eruptions, and the statistics of such shells in the Galaxy indicate that these eruptions are likely the dominant mass loss mechanism for evolved, $M_{Z A M S} \gtrsim 40 M_{\odot}$ stars. Dust formation at such high $\dot{M}$ also explains why very large grains $\left(a_{\max } \gtrsim 1 \mu \mathrm{m}\right)$ are frequently found in these shells, since $a_{\max } \propto \dot{M}$. The statistics of these shells (numbers, ages, masses, and grain properties such as $a_{\max }$ ) provide an archaeological record of this mass loss process. In particular, the velocities $v_{\text {shell }}$, transient durations (where known) and ejected masses $M_{\text {shell }}$ of the Galactic shells and the supernova "impostors" proposed as their extragalactic counterparts are very different. While much of the difference is a selection effect created by shell lifetimes $\propto\left(v_{\text {shell }} \sqrt{M_{\text {shell }}}\right)^{-1}$, more complete Galactic and extragalactic surveys are needed to demonstrate that the two phenomena share a common origin given that their observed properties are essentially disjoint. If even small fractions $(1 \%)$ of $\mathrm{SNe}$ show interactions with such dense shells of ejecta, as is currently believed, then the driving mechanism of the eruptions must be associated with the very final phases of stellar evolution, suggestive of some underlying nuclear burning instability.
\end{abstract}

Subject headings: stars: evolution; stars: massive; stars: mass-loss; supernovae: general; stars: winds, outflows; dust; extinction

\section{Introduction}

The winds of hot stars do not form dust, as illustrated by the single uses of the word "dust" in the reviews of these winds by Kudritzki \& Puls (2000) and Puls et al. (2008). The exceptions which prove the rule are the rare, dust forming WC stars, all of which appear to be binaries where dust forms due to the collision of the two stellar winds (see the review by Crowther 2007), and the relatively rare B[e] stars where dust is believed to form in a disk/dense equatorial wind surrounding the star (see the review by

\footnotetext{
${ }^{1}$ Department of Astronomy, The Ohio State University, 140 West 18th Avenue, Columbus OH 43210

${ }^{2}$ Center for Cosmology and AstroParticle Physics, The Ohio State University, 191 W. Woodruff Avenue, Columbus OH 43210
} 
Waters \& Waelkens 1998). Dust formation is inhibited by the low particle densities and the harsh ultraviolet (UV) environment (e.g. Cherchneff \& Tielens 1995). Cool star winds readily form dust, and there are extensive studies of dust formation in such environments (see the review by Willson 2000).

Yet it is clear the hot, massive stars can episodically form enormous quantities of dust and that this is related to the eruptions of luminous blue variables (LBVs, see the reviews by Humphreys \& Davidson 1994, Vink 2009). LBVs are observed in three states: a quiescent, hot $\left(T_{*}>15000 \mathrm{~K}\right)$ state, a cooler $\left(T_{*} \simeq\right.$ $7000 \mathrm{~K}$ ) eruptive or $\mathrm{S}$ Doradus state of roughly the same bolometric luminosity but enhanced mass loss rates ( $\dot{M} \sim 10^{-4}$ to $10^{-5} M_{\odot} /$ year), and a similarly cool, "great" eruptive state of significantly higher bolometric luminosity and enormously enhanced mass loss rates $\left(\dot{M} \gtrsim 10^{-2}-10^{-3} M_{\odot} /\right.$ year $)$. We will call these the hot (or quiescent), cool (or S Doradus), and (great) eruptive states, and we will refer to the ejected material from an eruption as a shell since the low duty cycles of eruptions produce relatively thin dusty shells of ejecta. In their (great) eruptive state, these stars can expel enormous amounts of material under conditions favorable to the growth of dust grains, as illustrated by the massive $\left(\sim 10 M_{\odot}\right.$, Smith et al. 2003), optically thick, dusty shell surrounding $\eta$ Carinae (see the reviews by Davidson \& Humphreys 1997, Smith 2009). Indeed, it is likely that such phases represent the bulk of the mass loss from higher mass stars $\left(M \gtrsim 40 M_{\odot}\right)$ because normal winds are inadequate to the task (Humphreys \& Davidson 1984, Smith \& Owocki 2006a). The recent discovery of many $24 \mu \mathrm{m}$ shells surrounding hot stars by Wachter et al. (2010) and Gvaramadze et al. (2010) further suggests that the phenomenon is more common than previously thought.

Table 1 summarizes the properties of Galactic LBVs and LBV candidates mainly drawn from Humphreys \& Davidson (1994) and Smith \& Owocki (2006a). All the stars have substantial, $\dot{M} \sim 10^{-5} M_{\odot} /$ year, relatively fast, $v_{\infty} \simeq 200 \mathrm{~km} / \mathrm{s}$, present day winds that are not forming dust, as expected for hot stellar winds. However, all but one system is surrounded by a relatively massive, $M_{\text {shell }} \sim M_{\odot}$, slowly expanding, $v_{\text {shell }} \sim 100 \mathrm{~km} / \mathrm{s}$, shell of dusty material, and in at least four cases, models of the shell appear to require surprisingly large maximum grain sizes, $a_{\max } \gtrsim 1 \mu \mathrm{m}$. We focused on these sources because most of these ancillary properties have been measured. Most of the mass estimates are based on assuming a dust-to-gas ratio $X_{d}=0.01$ and so could be underestimates.

While Table 1 is certainly incomplete and subject to many selection effects, that it contains 13 objects means that shell ejections are an important or even dominant mass loss process for massive stars, as has been previously suggested in order to compensate for the steady downward revisions of the mass loss rates in normal, hot stellar winds (e.g., Humphreys \& Davidson 1984, Smith \& Owocki 2006a). We can quantify this by estimating the number of dusty shells that should exist in the Galaxy given the rate of Galactic supernovae, $r_{S N}$. For simplicity, we use a Salpeter initial mass function (IMF) and assume that supernovae arise from stars with initial masses $M_{*}$ in the range $M_{S N} \simeq 8 M_{\odot} \lesssim M_{*} \lesssim M_{u p}$, where for now we will let $M_{u p} \rightarrow \infty$. If eruptions occur in stars with $M_{*}>M_{\text {erupt }}$ and there are an average of $N_{\text {erupt }}$ occurrences per star, then the eruption rate $r_{\text {erupt }}$ is of order

$$
r_{\text {erupt }} \simeq 0.1\left(\frac{40 M_{\odot}}{M_{\text {erupt }}}\right)^{1.35} N_{\text {erupt }} r_{S N} .
$$

The optical depth of a dusty shell of mass $M_{\text {shell }}$ with visual opacity $\kappa_{V} \simeq 100 \mathrm{~cm}^{2} / \mathrm{g}$ expanding at velocity 
$v_{\text {shell }}$ is $\tau_{V}=M_{\text {shell }} \kappa_{V} / 4 \pi v_{\text {shell }}^{2} t^{2}$ so the shell will be detectable for of order

$$
t_{\text {shell }} \simeq\left(\frac{M_{\text {shell }} \kappa_{V}}{4 \pi v_{\text {shell }}^{2} \tau_{V}}\right)^{1 / 2} \simeq 5700\left(\frac{0.1}{\tau_{V}}\right)^{1 / 2}\left(\frac{M_{\text {shell }}}{10 M_{\odot}}\right)^{1 / 2}\left(\frac{70 \mathrm{~km} / \mathrm{s}}{v_{\text {shell }}}\right) \text { years } .
$$

Most shells should be seen near their maximum size, $v_{\text {shell }} t_{\text {shell }} \simeq 0.4 \mathrm{pc}$, which is typical of the examples in Table 1. The total fraction of the stellar luminosity reradiated in the mid-IR is larger than $\tau_{V}$ because of the increased dust opacity in the UV. The expected number of Galactic shells is the product of the rate and the lifetime,

$$
N_{\text {shell }}=r_{\text {erupt }} t_{\text {shell }}=6 N_{\text {erupt }}\left(\frac{0.1}{\tau_{V}}\right)^{1 / 2}\left(\frac{r_{S N}}{\text { century }^{-1}}\right)\left(\frac{40 M_{\odot}}{M_{\text {erupt }}}\right)^{1.35}\left(\frac{M_{\text {shell }}}{10 M_{\odot}}\right)^{1 / 2}\left(\frac{70 \mathrm{~km} / \mathrm{s}}{v_{\text {shell }}}\right) .
$$

As we see from Table 1 , there are at least $N_{\text {shell }} \sim 10 \mathrm{LBV}$ stars surrounded by massive, dusty shells in the Galaxy, which means that the number of eruptions per star is

$$
N_{\text {erupt }} \simeq 2\left(\frac{N_{\text {shell }}}{10}\right)\left(\frac{\tau_{V}}{0.1}\right)^{1 / 2}\left(\frac{\text { century }^{-1}}{r_{S N}}\right)\left(\frac{M_{\text {erupt }}}{40 M_{\odot}}\right)^{1.35}\left(\frac{10 M_{\odot}}{M_{\text {shell }}}\right)^{1 / 2}\left(\frac{v_{\text {shell }}}{70 \mathrm{~km} / \mathrm{s}}\right) .
$$

and the amount of ejected mass per star due to the eruptions is $M_{\text {tot }}=N_{\text {erupt }} M_{\text {shell }} \simeq 15 M_{\odot}$. A "normal" wind from a hot star with $\dot{M}_{\text {normal }} \simeq 10^{-5} M_{\odot} /$ year would have to operate continuously for over $10^{6}$ years to equal the typical eruptive mass loss implied by the existence of even the well-studied Galactic shells. That $N_{\text {erupt }}>1$ is also consistent with the existence of multiple shells around some of the Galactic examples (e.g. G72.29+0.46, Jiménez-Esteban et al. 2010). Note, however, that the overall duty cycle of the shell phase is low, since $N_{\text {erupt }} t_{\text {shell }} \simeq 10^{4}$ years as compared to post-main-sequence lifetimes of order $10^{6}$ years. These estimates are broadly consistent with earlier estimates (e.g. Humphreys \& Davidson 1994, Lamers 1989) but based on a different approach.

Given such a large contribution to the mass loss history of massive stars, we need to understand the relationship between mass loss and dust formation around hot stars. We consider a parcel of fluid ejected in a wind of mass loss rate $\dot{M}$ and velocity $v_{w}$ ejected from a star of luminosity $L_{*}$ and temperature $T_{*}$ and examine the conditions under which dust can form in $\$ 2$. Not surprisingly, the key variable is the mass loss rate. First, for stellar winds with velocities of order the escape velocities of massive hot stars, very high mass loss rates are needed for particle growth. Second, the dust formation region must be shielded from the hot stellar photosphere, which these high density winds can achieve by forming a pseudo-photosphere in the wind with a characteristic temperature of roughly $7000 \mathrm{~K}$. Dust formation around hot blue stars is necessarily tied to very high mass loss rates, the classic LBV eruptive state and the formation of shells of ejecta. In $\$ 3$ we discuss some implications of this model for dust formation, stellar evolution and supernovae.

\section{The Physics of Dust Formation in Stellar Transient Ejecta}

We consider the formation of dust grains of radius $a$ comprised of $N$ atoms of average mass $m_{0}$ where $4 \pi a^{3} \rho_{\text {bulk }} / 3=N m_{0}$ and $\rho_{\text {bulk }}$ is the bulk density of the grain. We will use $\rho_{\text {bulk }}=2.2 \mathrm{~g} / \mathrm{cm}^{3}\left(3.8 \mathrm{~g} / \mathrm{cm}^{3}\right)$ and 


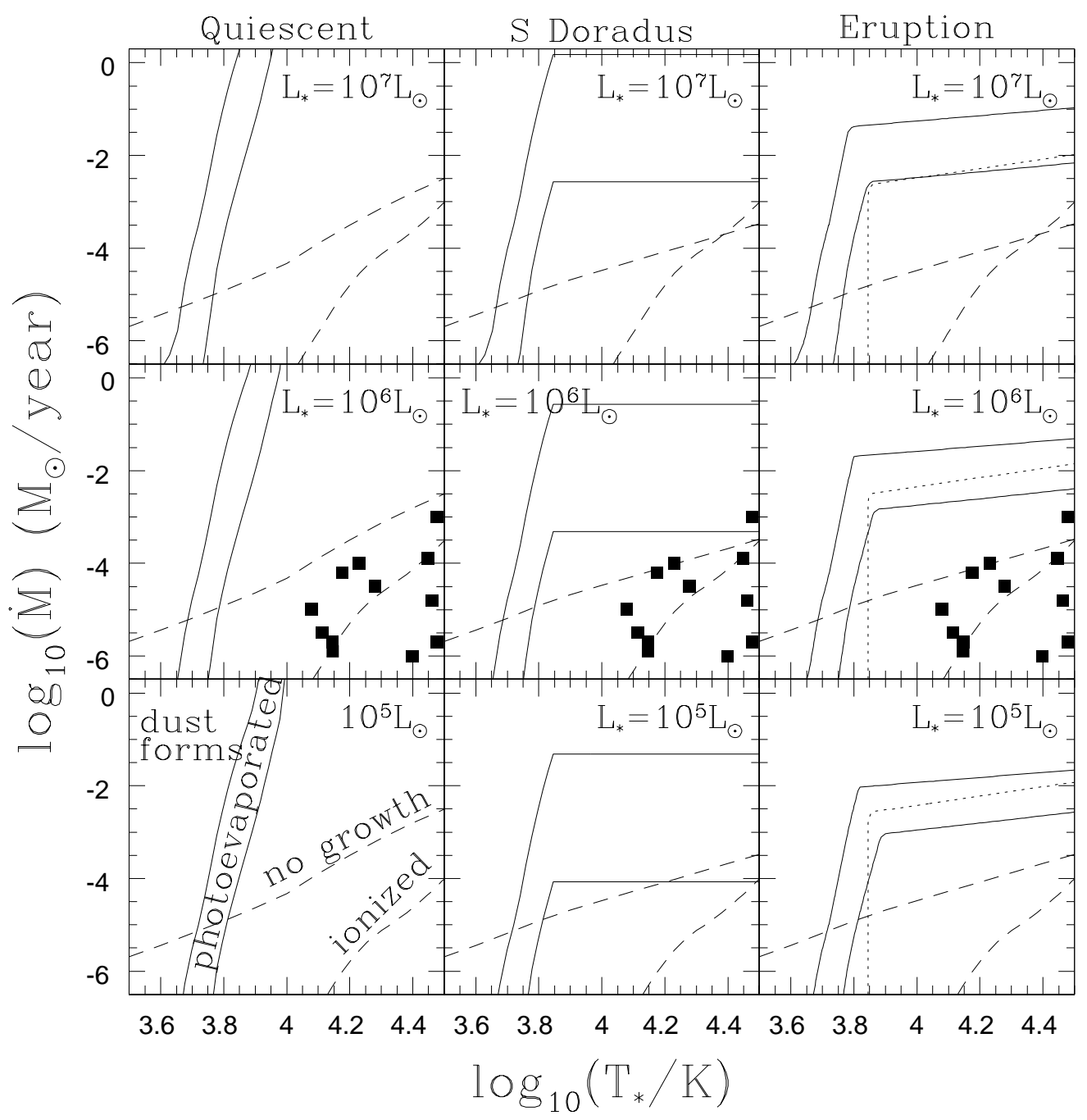

Fig. 1. - Minimum mass loss rates $\dot{M}$ for silicate dust formation as a function of stellar temperature $T_{*}$. The results for graphitic dusts are very similar. The lower left panel labels the regions. Dust can form in the region labeled "dust forms", above the photoionization ("ionized"), growth ("no growth") and photoevaporation ("photoevaporated") limits. The photoevaporation limits are for minimum photon energies of $E_{0}=7.5$ (stronger) and $10 \mathrm{eV}$ (weaker). The left, middle and right columns are for the three different assumptions about the apparent photospheric temperature. In the quiescent state (left) the photospheric temperature is the stellar temperature, $T_{p h o t}=T_{*}$, in the $\mathrm{S}$ Doradus state (middle) the photospheric temperature of $T_{\text {phot }}=\min \left(T_{*}, 7000 \mathrm{~K}\right)$ is assumed to be determined by an expansion of the stellar photosphere that is uncorrelated with the wind, and in the eruptive state (right) the apparent temperature is determined by the non-dust optical depth of the wind. The dotted contour in this column is the contour where $T_{p h o t}=7000 \mathrm{~K}$. The upper, middle and lower panels show the changes for stellar luminosities of $L_{*} \equiv L_{p h o t}=10^{7}, 10^{6}$ and $10^{5} L_{\odot}$, respectively. The filled points are the present day properties of the systems from Table 1 . Other parameters are set to $M_{*}=20 M_{\odot}, v_{c}=1 \mathrm{~km} / \mathrm{s}, X_{g}=0.005, T_{d}=1500 \mathrm{~K}, N=7, \hat{\rho}=3.8$ and $m_{0}=20 m_{p}$. 
$m_{0}=12 m_{p}\left(20 m_{p}\right)$ for graphitic (silicate) grains. The smallest possible grain is the point where the interparticle bond strengths shift from strong molecular bonds to weaker intra-molecular interactions, and we will generally require $N \geq 7$ based on the number of atoms in $\mathrm{Mg}_{2} \mathrm{SiO}_{4}$. There are many prior treatments of dust formation in (generally cool) stellar winds (e.g. Salpeter 1977, Draine 1979, Gail et al. 1984), novae (see the review by Gehrz 1988) and supernovae (e.g. Clayton 1979, Dwek 1988, Kozasa et al. 1991) which contain most of the basic physical picture we use here. For some standard results in dust physics we will refer to Draine (2011) as a reference source. For dust to grow, the medium must be largely neutral, sufficiently cool for growth to occur, have a high enough density for there to be an appreciable particle collision rate, and the grains must grow faster than they can be photo-evaporated by ultraviolet (UV) photons. We will assume that the first stage of particle formation, nucleation to form the smallest grains, simply occurs once the temperature is sufficiently low, and consider only the subsequent collisional growth of the grains. Making nucleation an additional bottleneck to dust formation will only strengthen our conclusions.

The dust forms in a (time varying) wind which we can characterize by the mass loss rate $\dot{M}$, the wind velocity $v_{w}$, and the mass fraction of condensible species $X_{g}$. Not all the condensible mass need condense onto grains, so the ultimate mass fraction of dust $X_{d} \leq X_{g}$. The wind is produced by a star of luminosity $L_{*}$, photospheric radius $R_{*}$ and effective temperature $T_{*}$ where $L_{*}=4 \pi R_{*}^{2} \sigma T_{*}^{4}$. At the time the dust is being formed, the star has luminosity $L_{p h o t}$, radius $R_{p h o t}$ and temperature $T_{p h o t}$, with $L_{p h o t}=4 \pi R_{p h o t}^{2} \sigma T_{p h o t}^{4}$. We consider dust formation in three physical states. First, we have the hot star in quiescence with $L_{p h o t}=L_{*}$ and $T_{p h o t}=T_{*}$. Second, we have the S Doradus state, where $L_{p h o t}=L_{*}$ and $T_{p h o t} \equiv 7000 \mathrm{~K} \neq T_{*}$. Finally, we have the eruptive state where both $L_{p h o t} \neq L_{*}$ and $T_{p h o t} \neq T_{*}$ and we will use the optical depth of the wind to estimate $T_{\text {phot }}$. In many cases we can assume that the spectral energy distribution is simply a black body, but there are several areas where the differences between black bodies and stellar photospheres are important because line opacities suppress the ultraviolet emission. Where this is important, we will use the models of Castelli \& Kurucz (2003).

For the physics of dust formation, the most relevant velocity is that near the dust formation radius since it sets the particle density that determines the growth of the grains. For hot stars, dust formation occurs sufficiently far from the star that the wind acceleration should be largely complete and we can view the velocity as constant. We will scale the wind velocity by the surface escape velocity of the quiescent stars

$$
v_{e}=\left(\frac{2 G M_{*}}{R_{*}}\right)^{1 / 2}=151\left(\frac{M_{*}}{20 M_{\odot}}\right)^{1 / 2}\left(\frac{10^{6} L_{\odot}}{L_{*}}\right)^{1 / 4}\left(\frac{T_{*}}{10^{4} \mathrm{~K}}\right) \mathrm{km} / \mathrm{s},
$$

as this is the typical asymptotic velocity scale of radiatively accelerated winds (see the reviews by Kudritzki \& Puls 2000, Puls et al. 2008). Here we have scaled the stellar mass to $M_{*}=20 M_{\odot}$ under the assumption that the stars have undergone significant mass loss by the time of the eruption. Since $v_{e} \propto T_{*}$, this introduces a strong stellar temperature dependence to dust formation and growth. In most of our results, the wind velocity is always scaled by the escape velocity of the quiescent star! Eqn. [5 yields an appropriate velocity scale for $\eta$ Carinae but may be somewhat high for many of the sources in Table 1. For the more massive examples in Table 1 the low shell expansion velocities are almost certainly intrinsic because only a very high density in- 
terstellar medium (ISM) can significantly slow the expansion of these massive shells of material. 1 . Moreover, some of the systems have multiple shells where the inner shells have low velocities but cannot be interacting with the ISM or an older slower wind (e.g. P Cyg, Meaburn et al. 1996; G79.29+0.46 Jiménez-Esteban et al. 2010). Thus, it may be that some systems have asymptotic velocities significantly lower than those from the surface of the quiescent hot star - this can be approximated by replacing $L_{*}$ and $T_{*}$ by $L_{p h o t}$ and $T_{p h o t}$, which will change the scaling of the wind velocity to use the escape velocity from the photosphere of the transient.

Luminous hot stars produce large numbers of ionizing photons, and dust cannot form in such a hot, ionized medium. For a pure hydrogen wind, the wind can recombine if the rate of production of ionizing photons is less than $Q_{0}=\dot{M}^{2} \alpha_{B} / 4 \pi v_{w}^{2} m_{p}^{2} R_{*}$ (e.g. Fransson 1982). The production of ionizing photons is

$$
Q_{0}=\frac{L_{*}}{k T_{*}} F\left(G=x^{-1}, E_{1} / k T_{*}, \infty\right)
$$

where $E_{1}=13.6 \mathrm{eV}$, the dimensionless function is

$$
F\left(G, x_{0}, x_{1}\right)=\frac{\int_{x_{0}}^{x_{1}} G F_{\nu} d x}{\int_{0}^{\infty} F_{\nu} d x} \rightarrow \frac{15}{\pi^{4}} \int_{x_{0}}^{x_{1}} \frac{G x^{3} d x}{\exp (x)-1},
$$

where $x=h \nu / k T$ and the limit is that of a black body. Thus, the minimum mass loss rate for the wind to recombine is

$$
\begin{aligned}
\dot{M} & \gtrsim\left(\frac{8 \pi L_{*} G M_{*} m_{p}^{2} F}{k T_{*} \alpha_{B}}\right)^{1 / 2}\left(\frac{v_{w}}{v_{e}}\right) \\
& \simeq 2.1 \times 10^{-3} F^{1 / 2}\left(\frac{3 \times 10^{-13} \mathrm{~cm}^{3} / \mathrm{s}}{\alpha_{B}} \frac{L_{*}}{10^{6} L_{\odot}} \frac{10^{4} \mathrm{~K}}{T_{*}} \frac{M_{*}}{20 M_{\odot}}\right)^{1 / 2}\left(\frac{v_{w}}{v_{e}}\right) M_{\odot} / \text { year } .
\end{aligned}
$$

For a black body, $F \simeq\left(15 / \pi^{4}\right)(2+x(2+x) \exp (-x))$ where $x=158000 / T_{*}$, making it a small number unless the star is very hot $\left(F^{1 / 2}=0.0024\right.$ for a $T_{*}=10^{4} \mathrm{~K}$ black body $)$, and the line blanketing of stellar atmospheres reduces it still further. Fig. 1 1 shows this photoionization limit ("ionized") on $\dot{M}$ for forming dust based on the Castelli \& Kurucz (2003) model atmospheres - unless the star is very hot, ionization cannot prevent dust formation in dense winds. Even then, the photoionization limit is only important for the quiescent star.

If the gas is relatively neutral, and so can carry out chemical reactions to form molecules, the temperature must be low enough to aggregate the molecules into grains. In general, the dust temperature is controlled by the radiation field because collisional time scales are very much longer (see below). We can divide the effects of radiation into the equilibrium temperature and stochastic heating of small grains by

\footnotetext{
${ }^{1}$ Given the shell masses it is very hard to slow them down by large factors. Even slowing a $1 M_{\odot}$ shell by a factor of two (from 140 to $70 \mathrm{~km} / \mathrm{s})$ within an expansion radius of $0.5 \mathrm{pc}$ requires an ISM density of order $10^{2} \mathrm{~cm}^{-3}$. A dense $\left(\dot{M} \gtrsim 10^{-5} M_{\odot} /\right.$ year $)$, pre-existing slow wind from a red supergiant phase is a more promising means of having this much mass present, but the timing must be right and it still would not explain the slow multiple shell systems. Slowing a massive $\left(10 M_{\odot}\right)$, fast $(500 \mathrm{~km} / \mathrm{s})$ shell like that of $\eta$ Carinae down to $100 \mathrm{~km} / \mathrm{s}$ requires $40 M_{\odot}$ of material, and is essentially impossible.
} 
individual energetic photons which we discuss below. If the dust temperature is controlled by radiative heating, then dust can form once small grains will not be heated above the dust destruction temperature $T_{d}$. If we consider only the mean temperature of the grains, then dust can form outside radius (Draine 2011)

$$
\begin{aligned}
R_{\text {form }} & =\left(\frac{L_{\text {phot }} Q_{P}\left(T_{\text {phot }}, a_{\text {min }}\right)}{16 \pi \sigma T_{d}^{4} Q_{P}\left(T_{d}, a_{\min }\right)}\right)^{1 / 2}=\frac{R_{*}}{2}\left(\frac{L_{\text {phot }}}{L_{*}}\right)^{1 / 2}\left(\frac{T_{*}}{T_{d}}\right)^{2}\left(\frac{Q_{P}\left(T_{\text {phot }}, a_{\text {min }}\right)}{Q_{P}\left(T_{d}, a_{\text {min }}\right)}\right)^{1 / 2} \\
& =5.2 \times 10^{14}\left(\frac{L_{\text {phot }}}{10^{6} L_{\odot}}\right)^{1 / 2}\left(\frac{1500 \mathrm{~K}}{T_{d}}\right)^{2}\left(\frac{Q_{P}\left(T_{\text {phot }}, a_{\text {min }}\right)}{Q_{P}\left(T_{d}, a_{\text {min }}\right)}\right)^{1 / 2} \mathrm{~cm}
\end{aligned}
$$

where $Q_{P}\left(T, a_{\min }\right)$ is the Planck-averaged absorption efficiency for the smallest grains. To simplify many subsequent results, we define

$$
Q_{\text {rat }} \equiv \frac{Q_{P}\left(T_{d}, a_{\min }\right)}{Q_{P}\left(T_{\text {phot }}, a_{\text {min }}\right)},
$$

so $R_{\text {form }}=\left(R_{*} / 2\right)\left(L_{\text {phot }} / L_{*}\right)^{1 / 2}\left(T_{*} / T_{d}\right)^{2} Q_{\text {rat }}^{-1 / 2}$. Note that the Planck factor for the star is evaluated at the apparent photospheric temperature $T_{p h o t}$ which may not be the same as the temperature at the stellar surface $T_{*}$. Unless $T_{d} \simeq T_{p h o t}$, the corrections for the finite size of the star are unimportant and for sufficiently small grains the result is independent of the grain size because the $Q \propto a$ dependence cancels. If we use the graphitic models of Draine \& Lee (1984) and $a_{\min }=0.001 \mu \mathrm{m}$, the Planck average for small graphitic dusts is approximately a power law

$$
Q_{P}(T)\left(\mu \mathrm{m} / a_{\min }\right) \simeq 0.42\left(\frac{T}{1000 \mathrm{~K}}\right)^{3 / 2}
$$

for $1000<T<50000 \mathrm{~K}$. Transient photospheric temperatures are generally $T_{\text {phot }} \simeq 7000 \mathrm{~K}$, so the formation radius for graphitic dusts is approximately

$$
R_{\text {form }} \simeq 1.6 \times 10^{15}\left(\frac{L_{\text {phot }}}{10^{6} L_{\odot}}\right)^{1 / 2}\left(\frac{1500 \mathrm{~K}}{T_{d}}\right)^{7 / 2} \mathrm{~cm} .
$$

The Planck averages for small silicate dusts cannot be reasonably approximated as a simple power law, but a reasonable piecewise approximation is

$$
\log _{10}\left[Q_{P}(T)\left(\mu \mathrm{m} / a_{\min }\right)\right] \simeq-0.66-0.34 t_{3}+1.15 t_{3}^{2} \text { for } 10^{3}<T<10^{4}
$$

where $t_{3}=\log _{10}(T / 1000 \mathrm{~K})$ and

$$
\log _{10}\left[Q_{P}(T)\left(\mu \mathrm{m} / a_{\min }\right)\right] \simeq 0.18+4.90 t_{4}-3.21 t_{4}^{2} \text { for } 10^{4}<T<5 \times 10^{4}
$$

where $t_{4}=\log _{10}(T / 10000 \mathrm{~K})$. For temperatures in the range $1000<T_{d}<2000 \mathrm{~K}$, the Planck averages vary little, so for silicate dusts and $T_{p h o t} \simeq 7000 \mathrm{~K}$ we find that

$$
R_{\text {form }} \simeq 9.8 \times 10^{14}\left(\frac{L_{\text {phot }}}{10^{6} L_{\odot}}\right)^{1 / 2}\left(\frac{1500 \mathrm{~K}}{T_{d}}\right)^{2} \mathrm{~cm} .
$$


In general, including the Planck factors makes the formation radius roughly three times larger than if they are ignored, and a reasonably general approximation is that $R_{\text {form }} \simeq 10^{15}\left(L_{\text {phot }} / 10^{6} L_{\odot}\right)^{1 / 2} \mathrm{~cm}$.

If we assume that particle nucleation occurs rapidly once the ejecta are cool enough to form dust, then the subsequent properties are limited by the growth of the dust particles. The collisional growth rate of a particle of radius $a$ is

$$
\frac{d a}{d t}=\frac{v_{c} X_{g} \dot{M}}{16 \pi v_{w} r^{2} \rho_{b u l k}}
$$

where $v_{c}$ is an effective collisional velocity (e.g. Kwok 1975, Deguchi 1980). For thermal collisions, accreting particles of mass $m_{a}$ at gas temperature $T$,

$$
v_{c}=\left(\frac{8 k T}{\pi m_{a}}\right)^{1 / 2}=4.6\left(\frac{T}{1000 \mathrm{~K}}\right)^{1 / 2}\left(\frac{m_{p}}{m_{a}}\right)^{1 / 2} \mathrm{~km} / \mathrm{s} .
$$

This means that growth cannot proceed by coagulation of large particles if the particle velocities are thermal because $m_{a}=N m_{0}$ means that $v_{c} \propto N^{-1 / 2}$ and the growth rate freezes out at very tiny grain sizes. In this case, growth must be dominated by the accretion of monomers and very small clusters, so we can regard $m_{a} \simeq m_{0}$ as effectively constant. Coagulation will matter if $v_{c}$ is controlled by turbulent motions (e.g. Voelk et al. 1980) with the net effect that particles can grow up to 4 times faster than by monomer accretion. The gas presumably cools as it expands, so we will let $v_{c}=v_{c 0}\left(R_{\text {form }} / R\right)^{n}$ where $R_{\text {form }}$ is the radius at which particle growth commences and $n=2 / 3$ if the cooling is dominated by adiabatic expansion and $n=1 / 4$ if it is controlled by radiative heating at constant luminosity. Other complications such as sticking probabilities and exhausting the condensible species can be mimicked by adjusting $v_{c 0}$ or $n$. With these assumptions, we find that the particles grow to a maximum size of

$$
\begin{aligned}
a_{\text {max }} & =\frac{v_{\text {cn }} X_{g} \dot{M}}{16 \pi \rho_{\text {bulk }} v_{w}^{2} R_{\text {form }}} \\
& \simeq 2.5 \times 10^{-4}\left(\frac{\dot{M} \hat{\rho}_{\text {bulk }}^{-1}}{10^{-4} M_{\odot} / \text { year }}\right)\left(\frac{X_{g}}{0.005}\right)\left(\frac{v_{c n}}{\mathrm{~km} / \mathrm{s}}\right)\left(\frac{500 \mathrm{~km} / \mathrm{s}}{v_{w}}\right)^{2}\left(\frac{10^{15} \mathrm{~cm}}{R_{\text {form }}}\right) \mu \mathrm{m} \\
& =\frac{v_{c n} X_{g} \dot{M}}{16 \pi G M_{*} \rho_{\text {bulk }}}\left(\frac{L_{*}}{L_{\text {phot }}}\right)^{1 / 2}\left(\frac{v_{e}}{v_{w}}\right)^{2}\left(\frac{T_{d}}{T_{*}}\right)^{2} Q_{\text {rat }}^{1 / 2} \\
& \simeq 5.3 \times 10^{-3}\left(\frac{\dot{M} \hat{\rho}_{\text {bulk }}^{-1} Q_{\text {rat }}^{1 / 2}}{10^{-4} M_{\odot} / \text { year }}\right)\left(\frac{X_{g}}{0.005}\right)\left(\frac{20 M_{\odot}}{M_{*}}\right)\left(\frac{v_{c n}}{\mathrm{~km} / \mathrm{s}}\right)\left(\frac{L_{*}}{L_{\text {phot }}}\right)^{1 / 2}\left(\frac{v_{e}}{v_{w}}\right)^{2}\left(\frac{T_{d}}{1500 \mathrm{~K}} \frac{10^{4} \mathrm{~K}}{T_{*}}\right)^{2} \mu \mathrm{m}
\end{aligned}
$$

where $v_{c n}=v_{c 0} /(1+n)$ absorbs the effects of the cooling model on $a_{\max }$ and $\hat{\rho}_{\text {bulk }}=\rho_{\text {bulk }} / \mathrm{g} / \mathrm{cm}^{3}$. The grain size grows with radius as

$$
a=a_{\max }\left(1-\frac{R_{\text {form }}^{1+n}}{R^{1+n}}\right) .
$$

Because the density is already dropping rapidly, reasonable assumptions about the temperature scaling $n$ have little effect on the results. Faster cooling leads to smaller particles, but the full range from a constant temperature to adiabatic cooling reduces $a_{\max }$ by less than a factor of two, and the particles are close to their final sizes by the time $R \simeq 2 R_{\text {form }}$. 
If the mass loss rates are too low, then the particles cannot grow, which implies there is a minimum mass loss rate for dust growth of

$$
\begin{aligned}
\dot{M} & >\frac{16 \pi G M_{*} \rho_{\text {bulk }}}{v_{c} X_{g}}\left(\frac{3 N m_{0}}{4 \pi \rho_{\text {bulk }}}\right)^{1 / 3}\left(\frac{v_{w}}{v_{e}}\right)^{2}\left(\frac{L_{\text {phot }}}{L_{*}}\right)^{1 / 2}\left(\frac{T_{*}}{T_{d}}\right)^{2} Q_{\text {rat }}^{-1 / 2} \\
& \gtrsim 3.2 \times 10^{-6}\left(\frac{\mathrm{km} / \mathrm{s}}{v_{c}}\right)\left(\frac{0.005}{X_{g}}\right)\left(\frac{v_{w}}{v_{e}}\right)^{2}\left(\frac{M_{*}}{20 M_{\odot}}\right)\left(\frac{L_{\text {phot }}}{L_{*}}\right)^{1 / 2}\left(\frac{1500 \mathrm{~K}}{T_{d}} \frac{T_{*}}{10^{4} \mathrm{k}}\right)^{2} \frac{\left(\hat{\rho}^{2} N \hat{m}_{0}\right)^{1 / 3}}{Q_{\text {rat }}^{1 / 2}} \frac{M_{\odot}}{\text { year }}
\end{aligned}
$$

where we have phrased the limit in terms of the particle number $N$ rather than the size $a$ and used $\hat{m}_{0}=$ $m_{0} / 12 m_{p}$. Fig. 1 compares these limits from particle growth ("no growth") to those from photoionization. The limits from particle growth are always the more stringent. When the wind velocity has $v_{w} \sim v_{e}$, it is very difficult for hot stars to form dust because of the rapid increase in the wind velocity with stellar temperature, $v_{w} \propto T_{*}$. We note that the limit at low temperatures appears high compared to typical AGB stars (e.g. van Loon et al. 2005, Matsuura et al. 2009) primarily because the mass has been scaled to $M_{*}=20 M_{\odot}$ and $\dot{M} \propto M_{*}$.

In the interstellar medium, the temperatures of the smallest dust grains are stochastic because the absorption of individual photons can temporarily heat the grains to temperatures far higher than the equilibrium temperature predicted by the ambient radiation density (e.g. Draine \& Anderson 1985, Dwek 1986). This effect also plays a key role in the formation of dust by transients, but seems not to have been generally considered outside estimates of dust formation in the colliding wind environments of WC stars (e.g. Cherchneff \& Tielens 1995). Under the assumption that the ejecta must recombine in order to form dust, we are interested in photons with energies $E_{0}<E<E_{1} \simeq 13.6 \mathrm{eV}$ since the hard UV photons are absorbed near the base of the wind. A small grain absorbing a soft UV photon will be heated well above the equilibrium temperature and then lose particles before radiatively cooling. Grains cannot grow if this photoevaporation rate is faster than the collisional growth rate (Draine \& Salpeter 1979). We can estimate $E_{0}$ using the models of Guhathakurta \& Draine (1989) for stochastic dust heating as the energy at which the grain cooling time scale equals the time to lose an atom from the grain. This energy depends crucially on what we view as the smallest number of particles in a grain because the peak temperature increases for smaller particle sizes and the probability of losing an atom rises exponentially with the peak temperature. If we consider a single $\mathrm{Mg}_{2} \mathrm{SiO}_{4}$ molecule with $N=7$ atoms as the smallest grain, then we find $E_{1} \simeq 6 \mathrm{eV}(9.0 \mathrm{eV})$ if the grain starts from an equilibrium temperature of $1500 \mathrm{~K}(1000 \mathrm{~K})$. If, however, we view the smallest grain as consisting of two such silicate units with $N=14$, then $E_{1} \simeq 13.6 \mathrm{eV}(21.0 \mathrm{eV})$. In either case, if the transient produces too many soft UV photons, small grains will be destroyed by the radiation faster than they can grow. If we define the absorption efficiency by $Q=Q^{\prime}(a / \lambda)$, the rate at which such photons are absorbed is

$$
t_{\gamma}^{-1}=\frac{L_{p h o t} a^{3}}{4 r^{2} h c} F\left(G=Q^{\prime}, E_{0} / k T_{p h o t}, E_{1} / k T_{p h o t}\right)
$$

where the function $F$ is the same as for the estimate of the number of ionizing photons in Eqn. 7 but with $G=Q^{\prime}$ rather than $G=1 / x$. Dust can only grow once the evaporation rate is lower than the collisional growth 
rate (Eqn. 17), leading to a photoevaporation limit on the mass loss rate for dust formation of

$$
\begin{aligned}
\dot{M} & >\frac{v_{w}}{v_{c}} \frac{L_{p h o t} a m_{0}}{X_{g} h c} F \\
& =3100 F \frac{v_{w}}{v_{e}} \frac{\mathrm{km} / \mathrm{s}}{v_{c}} \frac{L_{p h o t}}{10^{6} L_{\odot}}\left(\frac{10^{6} L_{\odot}}{L_{*}}\right)^{1 / 4}\left(\frac{T_{*}}{10^{4} \mathrm{~K}}\right)\left(\frac{M_{*}}{20 M_{\odot}}\right)^{1 / 2}\left(\frac{0.005}{X_{g}}\right)\left(\frac{\hat{m}_{0}^{4} N}{\hat{\rho}}\right)^{1 / 3} \frac{M_{\odot}}{\text { year }} .
\end{aligned}
$$

The factor $\left(\hat{m}_{0}^{4} N / \rho\right)^{1 / 3} \simeq 2$ for $N \simeq 7$. The enormous difference between the photon and particle densities means that the possibility of dust formation is entirely controlled by the spectral energy distribution of the transient and the value of $E_{0}$. As with the recombination limits, the differences between black bodies and actual photospheres are crucial - the limits on $\dot{M}$ for black bodies are several orders of magnitude higher than those for the Castelli \& Kurucz (2003) models. As we see in Fig. 1) the photoevaporation limit on $\dot{M}$ is a wall blocking dust formation in the quiescent state independent of mass loss rate. Thus, dust can only form around hot stars if they do not appear to be hot when observed from the dust formation radius. These stars appear to have two means of achieving this - the S Doradus phase and (great) eruptions.

In the $\mathrm{S}$ Doradus phase, the luminosity of the star is little changed, $L_{p h o t} \simeq T_{*}$, but the stars have cooler photospheric temperatures, $T_{\text {phot }} \simeq 7000 \mathrm{~K}$. For our model of the $\mathrm{S}$ Doradus phase, we adopt the more common view that the lower temperature is due to a true expansion of the stellar photosphere rather than a "pseudo-photosphere" formed in a dense wind (see the discussion in Vink 2009). In the S Doradus state, the stars have fairly high mass loss rates, $\dot{M} \sim 10^{-5}-10^{-4} M_{\odot}$, and fast winds with $v_{\infty} \simeq v_{e}$, but they cannot be forming significant amounts of dust even though they satisfy the condition on photospheric temperature. With a dust optical depth of

$$
\tau_{V}=\frac{\dot{M} \kappa_{V}}{4 \pi v_{w} R_{\text {form }}} \simeq 3\left(\frac{\dot{M}}{10^{-4} M_{\odot} / \text { year }}\right)\left(\frac{\kappa_{V}}{100 \mathrm{~cm}^{2} / \mathrm{g}}\right)\left(\frac{10^{15} \mathrm{~cm}}{R_{\text {in }}}\right) \frac{v_{e}}{v_{w}}
$$

the stars would become bright, hot mid-IR sources and some would be heavily enshrouded by their own dust, yet neither phenomenon seems to be reported.

As we show in the middle panels of Fig. 1, where we simply set the apparent photospheric temperature to $T_{\text {phot }}=\min \left(T_{*}, 7000 \mathrm{~K}\right)$, the cooler temperature is not sufficient to allow dust formation given the typical mass loss rates. First, the particle growth rates are too low. Second, the photosphere is still producing enough soft UV photons that the smallest grains still tend to photo-evaporate faster than they can grow. This second limit is very sensitive to the minimum photon energy $E_{0}$ needed to photo-evaporate a grain, but for $E_{0}=10 \mathrm{eV}$ the limit is close to the limit for any particle growth, at roughly $\dot{M} \gtrsim 10^{-4} M_{\odot} /$ year, If we lower the minimum energy to $E_{0}=7.5 \mathrm{eV}$, the required minimum mass loss rate jumps enormously because we are counting photons on the rapidly falling blue side of the spectrum. Thus, while the precise limits are sensitive to the exact choices of $T_{p h o t}$ and $E_{0}$, the combination of slow growth and photoevaporation mean that dust cannot form in the $\mathrm{S}$ Doradus phase.

The final case we consider is a (giant) eruption where there is an increase in the bolometric luminosity $L_{\text {phot }}>L_{*}$, the apparent temperature is cooler, $T_{p h o t}<T_{*}$, as in the S Doradus phases, and the mass loss rates are much higher, $\dot{M}>10^{-2} M_{\odot} /$ year. For sufficiently dense winds, the dust formation region sees a pseudophotosphere created by the non-dust opacity of the wind rather than the hot stellar photosphere. Davidson 
(1987) explains this as a consequence of combining a dense wind with an opacity law that is falling rapidly with temperature in this temperature range. Consider the Rosseland mean optical depth

$$
\tau_{R}(R)=\int_{R}^{R_{3}} \rho \kappa_{R}(\rho, T) d R
$$

looking inwards from the radius $R_{3}$ where the gas temperature is $1000 \mathrm{~K}$ and dust formation may be possible at some interior radius $R$. If we combine the rapidly rising $\rho \propto r^{-2}$ density profile of the wind with a temperature regime where the opacity rises rapidly, then there will be a tendency to produce a pseudo-photosphere where $\tau_{R}(R)=1$ near that temperature. We computed the temperature $T_{w}\left(R\left(\tau_{R}=1\right)\right)$ at the radius where $\tau_{R}=1$ using the solar composition opacity models of Helling \& Lucas (2009), our standard wind density profile and assuming a temperature profile $T_{w}=T_{*}\left(R / R_{*}\right)^{-1 / 2}$. This is not a self-consistent wind model, but the results are insensitive to the assumptions because the opacity and optical depth increase so rapidly towards smaller radii in the wind. Fig. 1 1 shows the consequences of using this "pseudo-photospheric" temperature in determining the photo-evaporation limit rather than $T_{*}$, as well as the contour where $T_{w}\left(R\left(\tau_{R}=1\right)\right)=7000 \mathrm{~K}$.

The limits now have two branches. For small $\dot{M}$ or low stellar temperatures, the wind is optically thin, the observed temperature is simply the photospheric temperature and the photo-evaporation limits are unchanged. For high $\dot{M}$ and high temperatures, the wind becomes optically thick and the observed temperature is of order $7000 \mathrm{~K}$ with relatively weak dependencies on $\dot{M}$ and $v_{w}$ because of the steep slope of the opacity, as predicted by Davidson (1987). As expected from the arguments summarized by Vink (2009), the mass loss rates needed to form a pseudo-photosphere are higher than are typically found for the S Doradus phase. However, once $\dot{M} \gtrsim 10^{-2.5} M_{\odot} /$ year, the wind forms a pseudo-photosphere whose temperature slowly drops with increasing mass loss rate, which makes the photevaporation limits less sensitive to $E_{0}$ than in our $\mathrm{S}$ Doradus model. Note that in both the S Doradus and eruption models the photosphere must stay in its cool state long enough for the ejecta to reach the dust formation radius ( $\sim 1$ year, Eqn. 10) if dust is to form.

Once dust forms, the size distribution then controls the opacity,

$$
\kappa_{\lambda}=\frac{3 X_{d}}{4 \rho_{\text {bulk }}} \frac{\left\langle Q_{\lambda}\left(a_{\max }\right)\right\rangle}{a_{\max }}
$$

where the dimensionless function

$$
\left\langle Q_{\lambda}\left(a_{\max }\right)\right\rangle=\frac{a_{\max } \int_{0}^{a_{\max }} Q_{\lambda}(a) a^{2} \frac{d n}{d a} d a}{\int_{0}^{a_{\max }} a^{3} \frac{d n}{d a} d a}
$$

depends on the grain size distribution $d n / d a$, the dimensionless (absorption or scattering) cross section $Q_{\lambda}(a)$, and the fraction of the gas mass in condensed dust $X_{d} \leq X_{g}$. The function $\left\langle Q_{\lambda}\left(a_{\max }\right)\right\rangle$ is proportional to $a_{\text {max }}$ for very small grains, where $Q_{\lambda} \propto a$ and becomes constant for very large grains where $Q_{\lambda}$ becomes constant (e.g. Draine \& Lee 1984). Thus, the ratio $\left\langle Q_{\lambda}\left(a_{\max }\right)\right\rangle / a_{\max }$ appearing in the opacity becomes constant for very small grains, decays as $a_{m a x}^{-1}$ for very large grains and has a maximum at an intermediate size $a_{\text {peak }}$. At $\mathrm{V}$ band for a Mathis et al. (1977) size distribution $d n / d a \propto a^{-3.5}$ with a range of $a_{\max } / a_{\min }=$ 50, we find $a_{\text {peak }} \simeq 0.16 \mu \mathrm{m}(0.45 \mu \mathrm{m})$ with $\left\langle Q_{\lambda}\left(a_{\text {peak }}\right)\right\rangle \simeq 2.4(\simeq 1.5)$ and $\left\langle Q_{\lambda}\left(a_{\text {peak }}\right)\right\rangle / a_{\text {peak }}=15.4 \mu \mathrm{m}^{-1}$ 
$\left(3.4 \mu \mathrm{m}^{-1}\right)$ for graphitic (silicate) dust. These estimates were made for the effective absorption optical depth $\left(\tau_{a b s}\left(\tau_{a b s}+\tau_{\text {scat }}\right)\right)^{1 / 2}$ and lead to maximum visual opacities of

$$
\begin{array}{rlr}
\kappa_{V, \text { max }} & \simeq 260 \frac{X_{d}}{0.005} \mathrm{~cm}^{2} / \mathrm{g} & \text { graphitic } \\
\kappa_{V, \max } \simeq 30 \frac{X_{d}}{0.005} \mathrm{~cm}^{2} / \mathrm{g} & \text { silicate }
\end{array}
$$

For $\tau_{a b s} / \tau_{\text {scat }}$ the coefficients are 210/13 and 130/75 for graphitic/silicate dusts. In general, however, the size dependence of the visual opacity is relatively weak. If $a_{\max } \gtrsim 1 \mu \mathrm{m}$ the opacity begins to drop as $a_{\max }^{-1}$ (Eqn. 21), but this requires $\dot{M} \gtrsim M_{\odot} /$ year, which no star seems to significantly exceed. For very small grains, $\left\langle Q_{\lambda}\left(a_{\max }\right)\right\rangle / a_{\max } \rightarrow 7.7 \mu \mathrm{m}^{-1}\left(0.6 \mu \mathrm{m}^{-1}\right)$ for graphitic (silicate) dust, so the opacity is only a factor of two (six) lower than the maximum opacity. If the grains cannot grow to moderate size, then the dust opacity will be significantly reduced.

\section{Discussion}

To summarize, the growth of dust particles in the ejecta of massive stars is limited by particle growth rates and photo-evaporation by soft, non-ionizing UV photons from the star. The particle growth rate is the limiting factor for lower temperature stars $\left(T_{*} \lesssim 7000 \mathrm{~K}\right)$, and photo-evaporation is the limiting factor for higher temperature stars $\left(T_{*} \gtrsim 7000 \mathrm{~K}\right)$. While they are hot stars in their quiescent state and cannot form dust in their winds, the LBVs have cooler apparent temperatures, $T_{p h o t} \simeq 7000 \mathrm{~K}$, in their S Doradus phases and during (great) eruptions. While they are cooler in the $\mathrm{S}$ Doradus phase, their mass loss rates are also not high enough for the grains to collisionally grow or to overcome the photoevaporation of small grains by the remaining soft UV photons. In this phase, the mass loss rates are not high enough for the non-dust opacity of the wind to self-shield the dust formation region, so the cooler temperature of the photosphere must be due to a true expansion of the photosphere, as argued in the review by Vink (2009). Only in (great) eruptions with mass loss rates $\dot{M} \gtrsim 10^{-2.5} M_{\odot}$ /year do these stars have the proper conditions for forming dust. Moreover, when the mass loss rates are this high, the wind does form a "pseudo-photosphere" with a temperature $T_{p h o t} \sim 7000 \mathrm{~K}$ that shields the dust formation region from the soft UV emission of the true stellar photosphere. This is the characteristic "eruption" temperature of luminous blue variables (e.g. Humphreys \& Davidson 1994) and it is a consequence of the steep rise of the non-dust opacity with temperature in this regime (Davidson 1987).

This distinction between an expanded photosphere for the S Doradus state and a "pseudo-photosphere" for the eruptions is also supported by the momentum transfers needed for a radiatively accelerated wind. Momentum conservation requires $\dot{M} v_{\infty} \simeq \tau L / c$, where $\tau$ is the non-dust opacity source responsible for accelerating the wind (see Kudritzki \& Puls 2000, Puls et al. 2008). For an asymptotic velocity of $v_{\infty}$, the optical depth must be

$$
\tau \simeq 10^{4}\left(\frac{\dot{M}}{M_{\odot} / \text { year }}\right)\left(\frac{10^{6} L_{\odot}}{L_{*}}\right) \frac{v_{\infty}}{v_{e s c}} .
$$

In the S Doradus phase, having $\dot{M} \lesssim 10^{-4} M_{\odot} /$ year and $v_{\infty} \simeq v_{e s c}$ means that $\tau<1$ and the wind cannot form 


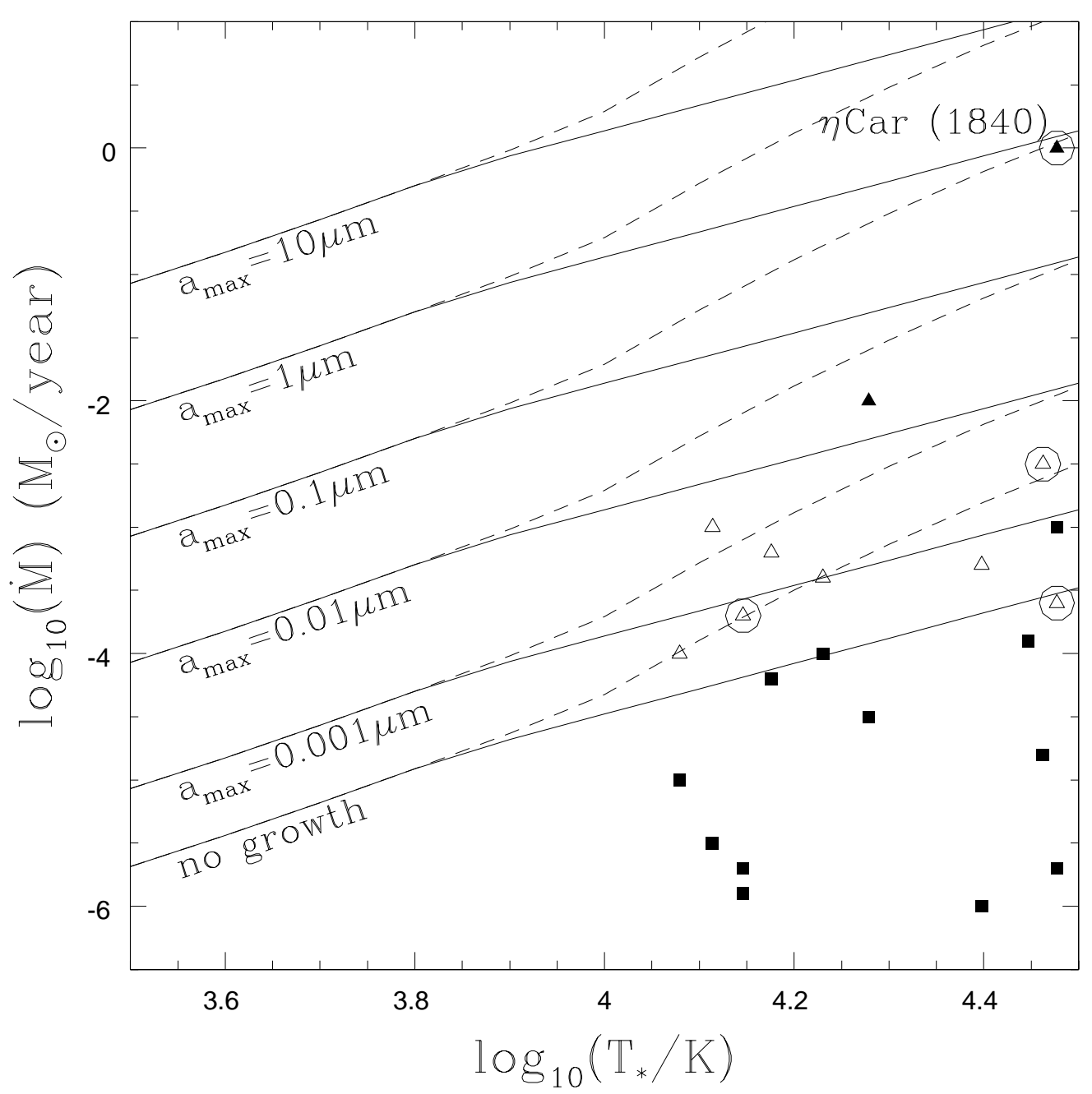

Fig. 2.- Mass loss rates needed to grow dust particles to radius $a_{\max }$ (Eqn. 21). In computing the ratio of Planck factors $Q_{r a t}$ we have either assumed the existence of a cooler photosphere $T_{p h o t}=\min \left(T_{*}, 7000 \mathrm{~K}\right)$ (solid) or used $T_{p h o t}=T_{*}$ (dashed). The triangles show the estimated mass loss rates during (great) eruptions based on either the observed duration (filled triangles, $\eta$ Car and P Cyg) or durations estimated from the shell widths (open triangles). We argue in the text that these latter estimates are gross underestimates of the mass loss rates in eruption. The filled squares show the present day properties of the systems in Table 1. Objects in Table 1 noted as having exceptionally large grain sizes are circled. The temperatures are left fixed at the present day temperature estimates - in reality they were cooler during the eruption but we lack direct measurements. 


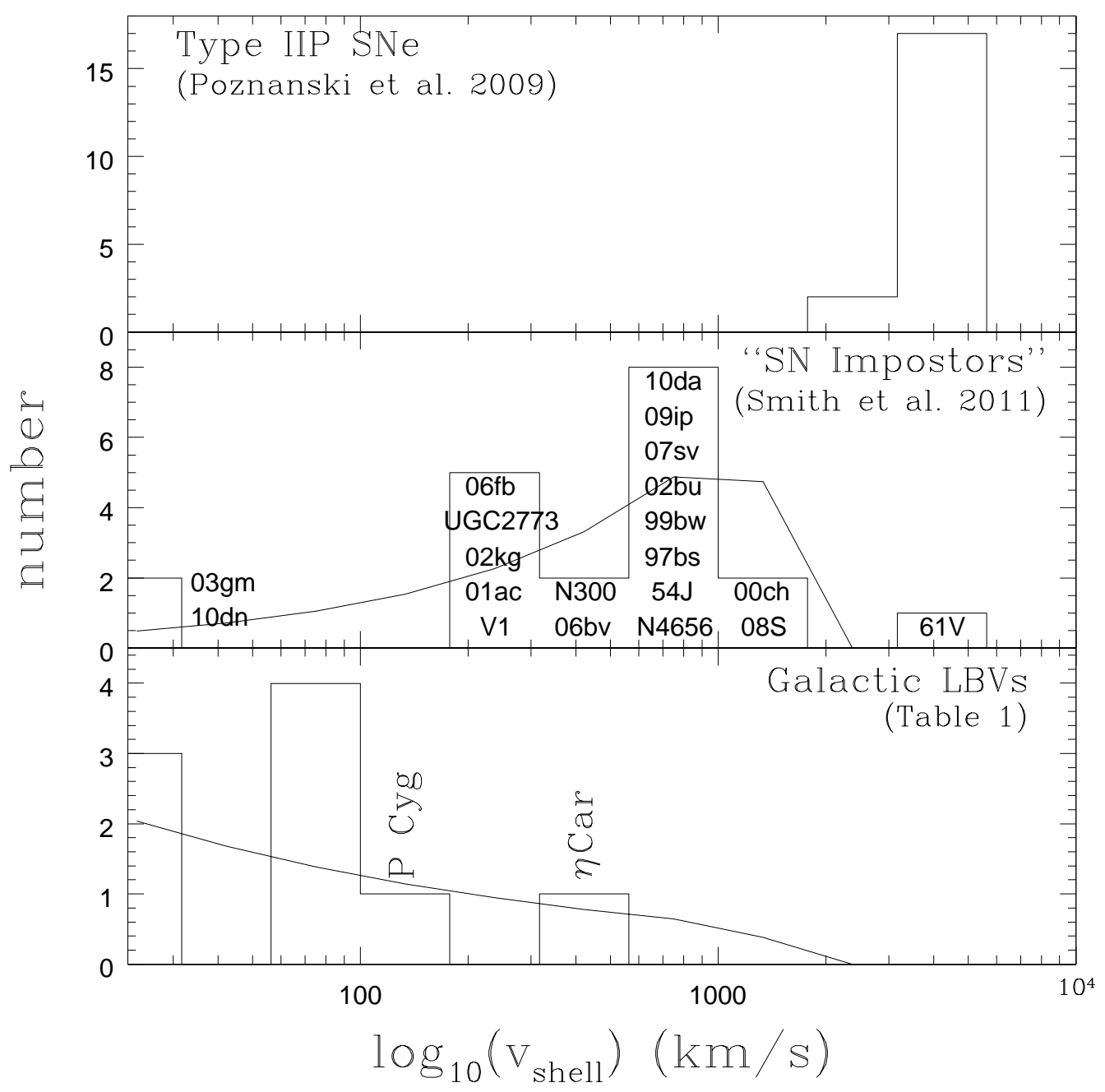

Fig. 3.- Asymptotic expansion velocities of Type IIP SNe (top), SN "impostors" (middle) and Galactic LBV shells (bottom). The impostor velocities have been corrected for expansion out of the stellar potential well following Eqn. 33 in order to properly compare them to the LBV shells. The general pattern of the results is not sensitive to the details of this correction. The $\mathrm{SNe}$ and the shells require no corrections because of their high velocities and ages, respectively. The curves in the impostor and Galactic LBV panels show the expected distributions for the bins assuming a velocity-dependent rate $r\left(v_{\text {shell }}\right) \propto v_{\text {shell }}^{-1 / 3}$ for $10 \mathrm{~km} / \mathrm{s}<$ $v_{\text {shell }}<1500 \mathrm{~km} / \mathrm{s}$ for the impostors and an observable lifetime $\propto 1 / v_{\text {shell }}$ for the Galactic shells normalized to the numbers of objects in each panel and excluding SN 1961V. 
a pseudo-photosphere. In the (great) eruptions with $\dot{M} \gtrsim 10^{-2} M_{\odot} /$ year, the optical depth must be $\tau \gg 1$ and the wind must have a pseudo-photosphere in order to be radiatively accelerated. We should also note that once dust dust forms, it can be a significant source of acceleration because the Eddington factor for radiation pressure on the dust,

$$
\Gamma_{d u s t}=\frac{\kappa_{r p} L_{p h o t}}{4 \pi c G M_{*}} \simeq 40\left(\frac{\kappa_{r p}}{100 \mathrm{~cm}^{2} / \mathrm{g}}\right)\left(\frac{L_{p h o t}}{10^{6} L_{\odot}}\right)\left(\frac{20 M_{\odot}}{M_{*}}\right),
$$

is large. Dust formation, as a large additional source of continuum opacity, may help to address some of the problems in accelerating these heavy winds (e.g. Owocki et al. 2004).

The first important consequence of this close relationship between dust formation and the need for very high mass loss rates is that the dust shells around luminous blue stars are formed exclusively in great eruptions and so trace the history of these eruptions. Given a census of such dusty shells, their radii, expansion velocities, (dust) masses and optical depths in the Milky Way or other galaxies, it should be possible to reconstruct this dominant mass loss mechanism for these massive stars. A particularly interesting diagnostic is the maximum grain size. Where the total dust mass or optical depth of a shell probes the total mass lost in the eruption, the maximum grain size probes the mass loss rate because, as shown in Fig. 2, the maximum grain size is proportional to the mass loss rate, $a_{\max } \propto \dot{M}$ (Eqn. 21). Models of four of the Galactic shells appear to require $a_{\max } \gtrsim 1 \mu \mathrm{m}$ (see Table 1 ) which strongly suggests $\dot{M} \gtrsim 10^{-2} M_{\odot} /$ year or possibly even higher.

A second consequence is that the mass loss rates associated with most of the shells in Table 1 are grossly underestimated - they are too low to make any dust let alone super-sized grains. These low estimates of $\dot{M}$ come from the assumption that the duration of the transient can be estimated from the radial thickness of the shell: $\Delta t \simeq \Delta R / v_{\text {shell }} \simeq 10^{4}$ years since $\Delta R \simeq R_{\text {shell }} \simeq 1 \mathrm{pc}$ and $v_{\text {shell }} \simeq 70 \mathrm{~km} / \mathrm{s}$. This leads to an estimate of $\dot{M}=10^{-4} M_{\odot} /$ year for $M_{\text {shell }}=M_{\odot}$ that is not very different from many of the present day winds which are not making dust, as illustrated in Fig. 2. The flaw here is that the observed spread in radius probably comes from temporal and azimuthal variations in velocity rather than the duration of the transient, just as we see in $\eta$ Carinae. This is proved by the simple geometric observation that all shells have comparable thickness ratios, which is the characteristic of a spread in velocity: $\Delta R=\Delta v t$ and $R=v t$ so $\Delta R / R=\Delta v / v$ is independent of time. If it were due to the duration of the transient then $\Delta R=v \Delta t$ and $R=v t$ so $\Delta R / R=\Delta t / t$ and the shells only appear geometrically thin as they become old. Roughly speaking, for every shell with a 2:1 thickness ratio there should be one which is a filled sphere just finishing its eruption, and this is not observed.

It should be possible to determine the geometric structure of these shells in some detail because many of the central stars are known to be significantly variable (e.g. $\eta$ Carinae, see, e.g., Fernández-Laiús et al. (2009) for a full light curve, or, e.g., Martin et al. 2006 for spatially resolved data; AG Car, Groh et al. 2009; IRAS 18576+3341, Clark et al. 2009). You can determine both the structure of the shell and obtain a geometric distance to the source by mapping the time delay between the variability of the star and the echoes of the variability across the shell, by essentially the same procedure as is used in reverberation mapping of quasars (see the review by Peterson 1993) or at a less involved level in studies of SN dust echoes (e.g. Patat 2005). This would complement the proper motion measurements possible for some systems (e.g. 
Smith et al. (2004a) for $\eta$ Carinae). The optimal wavelength is probably on the blue side of the mid-IR peak, at $10-20 \mu \mathrm{m}$ to maximize the sensitivity to dust temperature variations while minimizing the direct radiation from the star, but scattered optical or near-IR emission is another possibility if the central star is faint enough to allow imaging of the shell.

With the exception of the Great Eruption of $\eta$ Carinae $(250-500 \mathrm{~km} / \mathrm{s})$, the typical expansion velocities of the Galactic shells are only 50-100 km/s (see Table 1, Fig. 3). As we argued earlier in §2, these expansion velocities are unlikely to have been significantly slowed by decelerations due to sweeping up the surrounding interstellar medium and so must be associated with the ejection mechanism. The relatively low velocities of the Galactic shells mean that comparisons of the so-called "SN impostors" to LBV eruptions require detailed examination. Fig. 3 shows the expansion velocities of a sample of normal Type IIP SNe (Poznanski et al. 2009), the Galactic eruptions from Table 1, and the SN "impostors" from Smith et al. (2011). The latter have been conservatively corrected to an asymptotic expansion velocity at large radius by

$$
v_{\infty}^{2}=v^{2}-\frac{2 G M_{*}}{v t}
$$

where we used $M_{*}=40 M_{\odot}$ and $t=14$ days. While the corrections for some of the individual objects are sensitive to the choice of these parameters, the overall results are not. In this recasting of the similar figure from Smith et al. (2011), we see that almost none of the impostors have velocities similar to the Galactic shells. We must, however, exercise care in comparing the velocity distributions of impostors and Galactic shells in Fig. 3 because slowly expanding shells are detectable for longer periods of time, $t_{\text {shell }} \propto$ $1 / v_{\text {shell }}$ (Eqn. 2). If the intrinsic rate of eruptions with asymptotic velocities $v_{\text {shell }}$ is $r\left(v_{\text {shell }}\right)$, the number of observable Galactic shells is $\propto r\left(v_{\text {shell }}\right) / v_{\text {shell }}$, independent of any other consideration such as correlations between $v_{\text {shell }}$ and $M_{\text {shell }}$ or completeness.

Fig. 3 also shows a model for the velocity distributions that is consistent with both samples. We assumed the intrinsic rate as a function of asymptotic velocity is a power law, $r\left(v_{\text {shell }}\right) \propto v_{\text {shell }}^{\alpha}$ with $v_{\min }<$ $v_{\text {shell }}<v_{\max }$. We excluded SN 1961V since it was probably an SN (see Kochanek et al. 2011, Smith et al. 2011), but otherwise ignored other ambiguities as to the nature of the impostor sample (e.g. the very different physics of SN 2008S and the NGC 300-OT, see Kochanek 2011). The best fitting model has $\alpha \simeq-1 / 3$, $v_{\text {min }} \simeq 10 \mathrm{~km} / \mathrm{s}$ and $v_{\max } \simeq 1500 \mathrm{~km} / \mathrm{s}$. Given the numbers of objects, the uncertainties are large $(-0.75<$ $\alpha<0.15$ for an order of magnitude change in K-S test probabilities). If the true rate is $r\left(v_{\text {shell }}\right) \propto v_{\text {shell }}^{\beta}$, then the difference can be interpreted as a velocity-dependent completeness $c\left(v_{\text {shell }}\right) \propto v_{\text {shell }}^{\alpha-\beta}$. For example, if the true rate is independent of $v_{\text {shell }}(\beta=0)$, then either the impostor sample is incomplete at low velocities or the Galactic sample is incomplete at high velocities. There can be additional biases created by asymmetries in the ejection velocities, since the early time velocities may represent the fastest expanding material while the late time shell emission may be dominated by the slowest moving material - however, only some 50\% of the shells are strongly aspherical (?) and the factor of $\sim 2$ asymmetry in $\eta$ Carinae is not large enough to represent a significant bias.

We should note that velocity is not the only parameter in which there is essentially no overlap between the Galactic and impostor samples. First, the eruption time scales of the only two Galactic systems where they are known, $\eta$ Carinae and P Cyg, are an order of magnitude (or more) longer than those of almost all 
impostors (years to decades versus months, see Smith et al. 2011 for a summary), even though they are the only Galactic systems with relatively high velocities. Second, the ejected masses of the impostors almost certainly have to be far smaller than the typical Galactic shell. Assuming the impostors are radiatively driven, energy conservation means that the upper bounds on their ejected masses are $\lesssim 0.1 M_{\odot}$, while the typical Galactic shell has a mass $\gtrsim 1 M_{\odot}$. Like the velocity distribution, the mass differences can be driven by the lifetimes of the Galactic shells, $t_{\text {shell }} \propto M_{\text {shell }}^{-1 / 2}$ (Eqn. 2). Nonetheless, while the Galactic sources and the impostors may be produced by a single process with a broad parameter range (velocity, mass, time scale), the observed Galactic and extragalactic sources basically sample completely different regions of that parameter space. If these two populations are to be unified, then the statistics and properties of the local systems need to be systematically determined, and the completeness of the extragalactic surveys need to be improved.

In addition to (probably) being the dominant mass loss mechanism for massive stars, the eruptions may have a comparable importance to $\mathrm{SNe}$ as a source of dust, particularly as a source of large grains due to their favorable conditions for particle growth. Suppose that the final pre-SN mass of the stars undergoing eruptions is $M_{0}$ and that fraction $f$ of the lost mass is in eruptions producing material with a dust-to-gas ratio of $X_{d}$. For $\mathrm{SN}$ to dominate the dust production by massive stars, they must produce

$$
M_{d, S N} \gtrsim 0.09 f\left(\frac{X_{d}}{0.005}\right)\left(\frac{40 M_{\odot}}{M_{\text {erupt }}}\right)^{0.35}\left[1-0.26 \frac{M_{0}}{M_{\text {erupt }}}+0.26 \frac{M_{0}}{M_{\text {erupt }}}\left(\frac{M_{e}}{M_{\text {up }}}\right)^{0.35}-\left(\frac{M_{e}}{M_{\text {up }}}\right)^{0.35}\right] M_{\odot}
$$

of dust per SN. Unlike $\S 1$, we have allowed $M_{u p}$ to be finite. We know either from the arguments of Humphreys \& Davidson (1984) and Smith \& Owocki (2006a) or our estimates of shell statistics in $\S 1$ that $f \gtrsim 0.5$, so eruptions dominate the dust distribution unless $M_{d, S N} \gtrsim 0.02 f M_{\odot}$ if we are conservative $\left(M_{\text {erupt }}=\right.$ $\left.40 M_{\odot}, M_{0}=10 M_{\odot}, M_{u p}=100 M_{\odot}\right)$ or $M_{d, S N} \gtrsim 0.06 f M_{\odot}$ if we are more liberal $\left(M_{\text {erupt }}=20 M_{\odot}, M_{0}=5 M_{\odot}\right.$, $\left.M_{u p}=300 M_{\odot}\right)$. Moreover, the presence of a dense circumstellar medium may also enhance dust production by $\mathrm{SNe}$ (Smith et al. 2008a). While SN dust production rates are uncertain, few SNe show evidence for producing this amount of dust (see the discussion in Matsuura et al. 2009), although Matsuura et al. (2011) subsequently reported the detection of $0.4-0.7 M_{\odot}$ of dust associated with SN 1987A.

There is also increasing evidence that such high mass loss phases are crucial to understanding SNe on two levels. The first problem is simply one of rates. For the nominal parameters suggested by our discussion of the abundance of shells in $\S 1$, the rate of eruptions must be roughly the same as the rate of $\mathrm{SNe}$. However, the rates of the faint Type IIn SNe generally believed to correspond to such transients are significantly lower (e.g. Li et al. 2011). This is consistent with the arguments in Thompson et al. (2009) and Horiuchi et al. (2011) that there is strong evidence for incompleteness in $M_{V} \lesssim-16$ mag transients. Many candidate eruptions are significantly fainter, with $-10.5<M_{V}<-15 \mathrm{mag}$ (Smith et al. 2011), so the completeness of these surveys for eruptions is presumably still worse. Local surveys need to find these fainter transients in order to make a complete inventory. The second issue is that there appears to be a mismatch between massive star formation rates and SN rates of almost a factor of two (Horiuchi et al. 2011). If the underlying estimates of massive star formation rates and $\mathrm{SNe}$ rates are correct, then either many of these fainter transients need to be SNe or there must be a significant population of failed SN (Horiuchi et al. 
2011). This is another facet of the need to correctly classify the impostors in order to understand their statistics.

The final issue we consider is that some supernova show evidence in their evolution that they are interacting with the massive dense shells of material created by these eruptions. The most dramatic examples are the hyperluminous Type IIn SNe (Smith \& McCrav 2007), although these seem to be related to low metallicity environments (Kozłowski et al. 2010, Stoll et al. 2011). However, Fox et al. (2011) argue that many Type IIn SNe show evidence for CSM interactions requiring the dense shells produced by eruptions. More generally, the existence of a dust echo from an SNe implies an eruption within $10^{3}-10^{4}$ years whenever the progenitor is a hot star if our theory that dust only forms in eruptions is correct 2 In fact, some hyperluminous $\mathrm{SNe}$ shows evidence for the presence of two shells - an inner one to boost the total luminosity and an outer dusty shell (e.g. Smith et al. 2008b, Kozłowski et al. 2010). The existence of any such correlation has dramatic implications for the cause of stellar eruptions. In order to produce any strong SN interaction phenomena, the shell of material must have been produced within time $t_{i n t}=10^{1.5}-10^{2.5}$ years of the SN. Suppose fraction $f_{\text {int }} \simeq 10^{-2}-10^{-1}$ of SNe require the CSM densities of eruptions, then the time period $\Delta t$ prior to the $\mathrm{SNe}$ over which the ejections can be occurring is

$$
\Delta t=0.1 N_{\text {erupt }} \frac{t_{\text {int }}}{f_{\text {int }}}\left(\frac{40 M_{\odot}}{M_{\text {erupt }}}\right)^{1.35} \simeq 10^{4}\left(\frac{N_{\text {erupt }}}{2}\right)\left(\frac{t_{\text {int }}}{300 \text { years }}\right)\left(\frac{0.01}{f_{\text {int }}}\right)\left(\frac{40 M_{\odot}}{M_{\text {erupt }}}\right)^{1.35} \text { years. }
$$

The existence of dust echoes leads to a similar conclusion - while $t_{\text {int }}$ is larger, their incidence in SNe $f_{\text {int }}$ is higher. While this point has been made before in a qualitative sense (see, e.g. Smith et al. 2010, Smith et al. 2011), Eqn. 35 makes it quantitatively clear how strong a constraint results from the existence of any such correlation. Moreover, the parameters chosen for the scaling in Eqn. 35 may be significantly overestimating $\Delta t$. If any such correlation exists, massive shell ejections are forced to be associated with the very last phases of massive star evolution, roughly to the onset of carbon burning, and this suggests that the underlying driving mechanism is post-carbon ignition nuclear burning instabilities (see the discussion in Smith \& McCrav 2007). Unfortunately, the only known mechanism of this kind, the pair instability SN (Woosley et al. 2007), requires very high masses $\left(M_{*} \gtrsim 100 M_{\odot}\right)$ and should not function at the metallicities of any of these nearby examples. There could still be a strong metallicity effect because of the dependence of line-driven stellar winds on metallicity (see Puls et al. 2008). As mass loss by normal winds becomes less efficient, stars may be more dependent on eruptions for mass loss, although this begs the question of how eruptions might become more efficient at lower metallicity. This question could be addressed by the investigating the statistics of LBV eruptions and shells as a function of environment.

The author thanks K. Davidson, R. Humphreys, M. Pinsonneault, K. Sellgren, N. Smith, K.Z. Stanek, D.M. Szczygiel, T.A. Thompson and B.E. Wyslouzil for comments and discussions. C.S.K. is supported by National Science Foundation (NSF) grant AST-0908816

\footnotetext{
${ }^{2}$ This discussion does not apply to dust echoes from the SNe of red supergiants where it is feasible to produce the dust in a slow, steady wind.
} 
Table 1. Summary of Galactic LBVs With Dusty Shells

\begin{tabular}{|c|c|c|c|c|c|c|c|c|c|c|}
\hline Object & $\begin{array}{l}L_{*} \\
L_{\odot}\end{array}$ & $\begin{array}{l}T_{*} \\
\mathrm{~K}\end{array}$ & $\begin{array}{c}\dot{M}_{n o w} \\
M_{\odot} / \mathrm{yr}\end{array}$ & $\begin{array}{c}v_{w, \text { now }} \\
\mathrm{km} / \mathrm{s}\end{array}$ & $\begin{array}{c}R_{\text {shell }} \\
\mathrm{pc}\end{array}$ & $\begin{array}{l}v_{\text {shell }} \\
\mathrm{km} / \mathrm{s}\end{array}$ & $\begin{array}{c}M_{\text {shell }} \\
M_{\odot}\end{array}$ & $\begin{array}{l}\dot{M}_{\text {shell }} \\
M_{\odot} / \mathrm{yr}\end{array}$ & $\begin{array}{c}a_{\max } \\
\mu \mathrm{m}\end{array}$ & References \\
\hline$\eta$ Car (1840) & $10^{6.7}$ & 30000 & $10^{-3.0}$ & 500 & 0.08 & $250 / 500$ & 15 & $10^{0.0}$ & 1 & D97,S09 \\
\hline$\eta$ Car (1890) & & & & & 0.03 & $140 / 300$ & 0.1 & $10^{-2.0}$ & & \\
\hline Wray 17-96 & $10^{6.3}$ & 13000 & $10^{-5.5}$ & 100 & 1.0 & & 10 & $10^{-3.0}$ & & E02 \\
\hline AG Car & $10^{6.2}$ & 29000 & $10^{-4.8}$ & 110 & 0.80 & 70 & 25 & $10^{-2.5}$ & 10 & S91,L94,V00 \\
\hline G79.29+0.46 & $10^{6.1}$ & 25000 & $10^{-6.0}$ & 110 & 1.8 & 30 & 14 & $10^{-3.3}$ & & W96,V00b,J10 \\
\hline $\mathrm{G} 26.47+0.02$ & $10^{6.0}$ & 17000 & $10^{-4.0}$ & $\equiv 200$ & 2.3 & & 1.9 & $10^{-3.4}$ & & $\mathrm{C} 03$ \\
\hline P Cyg & $10^{5.9}$ & 19000 & $10^{-4.5}$ & 190 & 0.07 & 136 & 0.1 & $10^{-2.0}$ & & N01, S06 \\
\hline Wra 751 & $10^{5.8}$ & 30000 & $10^{-5.7}$ & $\equiv 500$ & 0.34 & 26 & 1.7 & $10^{-3.6}$ & 1 & H91b,deW92,V00 \\
\hline IRAS 18576 & $10^{5.8}$ & 15000 & $10^{-4.2}$ & 160 & 0.15 & 70 & 10 & $10^{-3.2}$ & & U01,U05,C09,B10 \\
\hline W 243 & $10^{5.8}$ & 18000 & $10^{-5.4}$ & & & & & & & $\mathrm{C} 04$ \\
\hline Hen 3-519 & $10^{5.7}$ & 28000 & $10^{-3.9}$ & 365 & 1.1 & 61 & 0.66 & & & $\mathrm{~S} 94$ \\
\hline HR Car & $10^{5.6}$ & 14000 & $10^{-5.7}$ & 145 & 0.3 & $30 / 100$ & 3.0 & & & H91a,L96,M02 \\
\hline $\mathrm{G} 24.73+0.69$ & $10^{5.6}$ & 12000 & $10^{-5.0}$ & $\equiv 200$ & 1.6 & & 0.5 & $10^{-4.0}$ & & $\mathrm{C} 03$ \\
\hline HD 168625 & $10^{5.4}$ & 14000 & $10^{-5.9}$ & 180 & 0.48 & 19 & 0.25 & $10^{-3.7}$ & 1 & N96,P02,003 \\
\hline
\end{tabular}

Note. - Luminosities and temperatures are from Smith et al. (2004b). Other sources are B10 (Buemi et al. 2010), C03 (Clark et al. 2003), C04 (Clark \& Negueruela 2004), C09 (Clark et al. 2009), D97 (Davidson \& Humphreys 1997), deW92 (de Winter et al. 1992), E02 (Egan et al. 2002), H91a (Hutsemekers \& van Drom 1991a), H91b (Hutsemekers \& van Drom 1991b), J10 (Jiménez-Esteban et al. 2010), L96 (Lamers et al. 1996), L94 (Leitherer et al. 1994), M02 (Machado et al. 2002), N01 (Najarro 2001), N96 (Nota et al. 1996), O03 (O'Hara et al. 2003), P02 (Pasquali et al. 2002), S91 (Smith 1991), S94 (Smith et al. 1994), S06 (Smith \& Hartigan 2006b), S09 (Smith 2009), V00a (Voors et al. 2000a), V00b (Voors et al. 2000b), U01 (Ueta et al. 2001), U05 (Umana et al. 2005), W96 (Waters et al. 1996). The ejected mass estimates are generally derived from the mid-IR dust luminosities and assume a dust-to-gas ratio of $X_{d}=0.01$. For $\eta$ Carinae the dust mass estimate agrees with estimates of the ejected gas mass (Smith \& Ferland 2007). The S06 estimate for P Cyg is a gas mass estimate. The dust content of P Cyg is uncertain, although there is a mid-IR excess (see S06). 


\section{REFERENCES}

Buemi, C. S., Umana, G., Trigilio, C., Leto, P., \& Hora, J. L. 2010, ApJ, 721, 1404

Castelli, F., \& Kurucz, R. L. 2003, Modeling of Stellar Atmospheres, 210, 20P

Cherchneff, I., \& Tielens, A. G. G. M. 1995, Wolf-Rayet Stars: Binaries; Colliding Winds; Evolution, 163, 346

Clark, J. S., \& Negueruela, I. 2004, A\&A, 413, L15

Clark, J. S., Egan, M. P., Crowther, P. A., Mizuno, D. R., Larionov, V. M., \& Arkharov, A. 2003, A\&A, 412, 185

Clark, J. S., Crowther, P. A., Larionov, V. M., Steele, I. A., Ritchie, B. W., \& Arkharov, A. A. 2009, A\&A, 507,1555

Clayton, D. D. 1979, Ap\&SS, 65, 179

Crowther, P. A. 2007, ARA\&A, 45, 177

Davidson, K. 1987, ApJ, 317, 760

Davidson, K., \& Humphreys, R. M. 1997, ARA\&A, 35, 1

Deguchi, S. 1980, ApJ, 236, 567

de Winter, D., Perez, M. R., Hu, J. Y., \& The, P. S. 1992, A\&A, 257, 632

Draine, B. T. 1979, Ap\&SS, 65, 313

Draine, B. T. 2011, Physics of the Interstellar and Intergalactic Medium by Bruce T. Draine. Princeton University Press, 2011. ISBN: 978-0-691-12214-4,

Draine, B. T., \& Salpeter, E. E. 1979, ApJ, 231, 438

Draine, B. T., \& Lee, H. M. 1984, ApJ, 285, 89

Draine, B. T., \& Anderson, N. 1985, ApJ, 292, 494

Dwek, E. 1988, ApJ, 329, 814

Dwek, E. 1986, ApJ, 302, 363

Egan, M. P., Clark, J. S., Mizuno, D. R., Carey, S. J., Steele, I. A., \& Price, S. D. 2002, ApJ, 572, 288

Fernández-Lajús, E., et al. 2009, A\&A, 493, 1093

Fox, O. D., et al. 2011, arXiv:1104.5012 
Fransson, C. 1982, A\&A, 111, 140

Gail, H.-P., Keller, R., \& Sedlmayr, E. 1984, A\&A, 133, 320

Gvaramadze, V. V., Kniazev, A. Y., \& Fabrika, S. 2010, MNRAS, 405, 1047

Gehrz, R. D. 1988, ARA\&A, 26, 377

Groh, J. H., Hillier, D. J., Damineli, A., Whitelock, P. A., Marang, F., \& Rossi, C. 2009, ApJ, 698, 1698

Guhathakurta, P., \& Draine, B. T. 1989, ApJ, 345, 230

Helling, C., \& Lucas, W. 2009, MNRAS, 398, 985

Horiuchi, S., Beacom, J. F., Kochanek, C. S., Prieto, J. L., Stanek, K. Z., \& Thompson, T. A. 2011, arXiv:1102.1977

Humphreys, R. M., \& Davidson, K. 1984, Science, 223, 243

Humphreys, R. M., \& Davidson, K. 1994, PASP, 106, 1025

Hutsemekers, D., \& van Drom, E. 1991, A\&A, 248, 141

Hutsemekers, D., \& van Drom, E. 1991, A\&A, 251, 620

Jiménez-Esteban, F. M., Rizzo, J. R., \& Palau, A. 2010, ApJ, 713, 429

Kochanek, C. S., Szczygiel, D. M., \& Stanek, K. Z. 2011, ApJ, 737, 76

Kochanek, C. S. 2011, arXiv:1106.4722

Kozasa, T., Hasegawa, H., \& Nomoto, K. 1991, A\&A, 249, 474

Kozłowski, S., et al. 2010, ApJ, 722, 1624

Kwok, S. 1975, ApJ, 198, 583

Kudritzki, R.-P., \& Puls, J. 2000, ARA\&A, 38, 613

Lamers, H. J. G. L. M. 1989, IAU Colloq. 113: Physics of Luminous Blue Variables, 157, 135

Lamers, H. J. G. L. M., et al. 1996, A\&A, 315, L225

Leitherer, C., et al. 1994, ApJ, 428, 292

Li, W., et al. 2011, MNRAS, 412, 1441

Meaburn, J., Lopez, J. A., Barlow, M. J., \& Drew, J. E. 1996, MNRAS, 283, L69

Machado, M. A. D., de Araújo, F. X., Pereira, C. B., \& Fernandes, M. B. 2002, A\&A, 387, 151 
Martin, J. C., Davidson, K., \& Koppelman, M. D. 2006, AJ, 132, 2717

Mathis, J. S., Rumpl, W., \& Nordsieck, K. H. 1977, ApJ, 217, 425

Matsuura, M., et al. 2009, MNRAS, 396, 918

Matsuura, M., et al. 2011, arXiv:1107.1477

Najarro, F. 2001, P Cygni 2000: 400 Years of Progress, 233, 133

Nota, A., Pasquali, A., Clampin, M., Pollacco, D., Scuderi, S., \& Livio, M. 1996, ApJ, 473, 946

O’Hara, T. B., Meixner, M., Speck, A. K., Ueta, T., \& Bobrowsky, M. 2003, ApJ, 598, 1255

Owocki, S. P., Gayley, K. G., \& Shaviv, N. J. 2004, ApJ, 616, 525

Pasquali, A., Nota, A., Smith, L. J., Akiyama, S., Messineo, M., \& Clampin, M. 2002, AJ, 124, 1625

Patat, F. 2005, MNRAS, 357, 1161

Peterson, B. M. 1993, PASP, 105, 247

Poznanski, D., et al. 2009, ApJ, 694, 1067

Puls, J., Vink, J. S., \& Najarro, F. 2008, A\&A Rev., 16, 209

Salpeter, E. E. 1977, ARA\&A, 15, 267

Smith, L. J. 1991, Wolf-Rayet Stars and Interrelations with Other Massive Stars in Galaxies, 143, 385

Smith, L. J., Crowther, P. A., \& Prinja, R. K. 1994, A\&A, 281, 833

Smith, N., Gehrz, R. D., \& Krautter, J. 1998, AJ, 116, 1332

Smith, N., Gehrz, R. D., Hinz, P. M., et al. 2003, AJ, 125, 1458

Smith, N., et al. 2004, ApJ, 605, 405

Smith, N., Vink, J. S., \& de Koter, A. 2004, ApJ, 615, 475

Smith, N., \& Hartigan, P. 2006, ApJ, 638, 1045

Smith, N., \& Owocki, S. P. 2006, ApJ, 645, L45

Smith, N., \& McCray, R. 2007, ApJ, 671, L17

Smith, N., \& Ferland, G. J. 2007, ApJ, 655, 911

Smith, N., Foley, R. J., \& Filippenko, A. V. 2008, ApJ, 680, 568

Smith, N., Foley, R. J., Bloom, J. S., et al. 2008, ApJ, 686, 485 
Smith, N. 2009, arXiv:0906.2204

Smith, N., Chornock, R., Silverman, J. M., Filippenko, A. V., \& Foley, R. J. 2010, ApJ, 709, 856

Smith, N., Li, W., Silverman, J. M., Ganeshalingam, M., \& Filippenko, A. V. 2011, MNRAS, 415, 773

Stoll, R., Prieto, J. L., Stanek, K. Z., Pogge, R. W., Szczygieł, D. M., Pojmański, G., Antognini, J., \& Yan, H. 2011, ApJ, 730, 34

Thompson, T. A., Prieto, J. L., Stanek, K. Z., Kistler, M. D., Beacom, J. F., \& Kochanek, C. S. 2009, ApJ, 705,1364

Ueta, T., Meixner, M., Dayal, A., Deutsch, L. K., Fazio, G. G., Hora, J. L., \& Hoffmann, W. F. 2001, ApJ, 548,1020

Umana, G., Buemi, C. S., Trigilio, C., \& Leto, P. 2005, A\&A, 437, L1

van Loon, J. T., Cioni, M.-R. L., Zijlstra, A. A., \& Loup, C. 2005, A\&A, 438, 273

Vink, J. S. 2009, in Eta Carinae and the Supernova Impostors, R. Humphreys \& K. Davidson, eds. (Springer) [arXiv:0905.3338]

Voelk, H. J., Jones, F. C., Morfill, G. E., \& Roeser, S. 1980, A\&A, 85, 316

Voors, R. H. M., et al. 2000, A\&A, 356, 501

Voors, R. H. M., Geballe, T. R., Waters, L. B. F. M., Najarro, F., \& Lamers, H. J. G. L. M. 2000, A\&A, 362, 236

Wachter, S., Mauerhan, J. C., Van Dyk, S. D., Hoard, D. W., Kafka, S., \& Morris, P. W. 2010, AJ, 139, 2330

Waters, L. B. F. M., \& Waelkens, C. 1998, ARA\&A, 36, 233

Waters, L. B. F. M., Izumiura, H., Zaal, P. A., Geballe, T. R., Kester, D. J. M., \& Bontekoe, T. R. 1996, A\&A, 313, 866

Weis, K. 2001, Reviews in Modern Astronomy, 14, 261

Willson, L. A. 2000, ARA\&A, 38, 573

Woosley, S. E., Blinnikov, S., \& Heger, A. 2007, Nature, 450, 390 